\title{
Complete colonic duplication in an infant
}

\author{
P D R Sisil Kumara', G L D P Jayawardane ${ }^{2}$ and A P R Aluwihare ${ }^{3}$
}

(Index words: Management, risk of malignancy).

\section{Introduction}

Both cystic and tubular varieties of colonic duplications are rare. We report a child with complete colonic duplication and three appendices, presenting with intestinal obstruction. The challenge in this instance was to devise an approach with minimal morbidity and maximal protection against risk of later malignancy in the duplication.

\section{Case report}

A 10-month old boy was referred by a general surgeon who found a long tubular colonic duplication when an exploratory laparotomy was done for acute intestinal obstruction. Only the obstructed duplication had needed decompression, done by bringing out 'three appendices' as appendicostomies in the right iliac fossa.

A second exploratory laparotomy in our unit revealed duplication of entire colon up to the lower rectum. The terminal ileum was seen to enter the normal large bowel and the three parallel appendices were attached to the duplication, which was on the anterior side. We found that the duplication did not communicate with the normal bowel at the distal end by injecting saline into its distal end while it was clamped proximally. A distal communication was created by inserting a long crushing clamp via the anus with one limb in the rectum and the other limb in the duplication through an opening made into it via the rectum, to crush the septum between the normal bowel and duplication, so that the duplication would drain distally in to the rectum

We converted the three appendicular lumina into one by crushing the septa between them. We then planned to close the common opening. However, contrast studies done at this time showed pooling of contrast in the right iliac fossa and no significant drainage distally. Hence we could not close the appendicostomy.

A third laparotomy was done when the child was 21 months old. We found that crushing of appendicular walls had not extended up to their bases. The large bowel looked like one tube with no external evidence of duplication. The caecal area was distended, and since it was not clear whether this would drain adequately, a right hemicolectomy was done including the appendicostomies. When the bowel was transected at the transverse colon, a longitudinal septum dividing the duplication from the normal bowel was seen and this was excised intra-luminally as far distally as possible before anastamosing the ileum to the common channel thus created. The duplication drained freely into the rectum via the previously established opening. The child made an uneventful recovery. The lining of the resected duplication did not contain ectopic mucosa. Since this child did not have abnormal genitalia or urinary symptoms and signs, we did not investigate his urinary system in detail.

\section{Discussion}

Colonic duplications occur along the mesenteric border of the intestine, sharing the muscle wall and blood supply with the "normal" bowel $(1,2,3)$. Some cases are associated with genitourinary anomalies including duplication of urethra or bladder $(1,2,3)$. The pathogenesis of these duplications has not been established. Theories include failure of regression of embryonic diverticula, median septum formation, partial twinning and errors of epithelial recanalisation $(1,2,3)$. Most duplications are found during infancy $(2,3)$. The presentations include an abdominal mass, constipation, intestinal obstruction, intussusception, volvulus and peritonitis due to perforation $(1,2,3,4,5)$

Colonic duplications are usually lined by colonic mucosa $(2,5)$, which has malignant potential $(3,4,5)$. There are reports of both squamous and adenocarcinoma arising in colonic duplications in adults $(5,6)$. Treatment should ideally seek to correct the presenting problem and minimise the risk of later malignancy.

Complete excision of the duplication is the treatment of choice $(2,3)$. However, this often requires excision of a similar length of the normal bowel because the shared blood supply and muscle wall $(1,2)$. In this patient a total colonic excision and ileo-rectal anastomosis would have been extensive surgery. The rare possibility of ectopic gastric mucosa being present in the duplication $(3,4,5)$ was not considered in this child as he did not have evidence of bleeding. Our initial approach was to achieve decompression of the duplication without extensive bowel resection.

Internal drainage of the duplication is one accepted option in these circumstances $(1,2,4)$. The disadvantage is that the entire mucosal lining of the duplication is left intact, and the child needs to be kept under long term observation to detect malignant transformation early.

Our attempt at internal drainage of the duplication failed initially, as there was poor antegrade drainage from the proximal part of the duplication, although the distal part was satisfactorily drained via the transrectal fenestration.

\footnotetext{
${ }^{12}$ Senior Paediatric Surgical Registrar, Teaching Hospital Peradeniya, ${ }^{3}$ Professor of Surgery, Faculty of Medicine, University of Peradeniya. (Accepted 18 November 2000).
} 
our next choice was resection of the proximal part of the duplication. Partial resection and mucosal stripping of the rest of the duplication would remove the entire mucosa $(1,2)$, but we did not favour this difficult procedure as it risked bowel injury.

We hope that the combination of drainage and passage of stool via the "duplication" might reduce the risk of malignancy. There are no data on this. One patient as young as 26 years developed cancer in a duplication (6), even though all patients reviewed in another study (5) were over $\mathbf{4 0}$ years of age when they presented with cancer in large bowel duplication. We plan colonoscopic surveillance of the remaining duplication in this child to detect malignant transformation early. We plan to start colonoscopy and serial biopsy from the mucosa of the duplication at the age of 25 years and repeat this every 5 years. If dysplasia is detected we will offer complete colectomy and ileo-rectal anastomosis.

In the light of our limited experience and previous reports, we recommend either internal drainage alone or partial resection with internal drainage as a satisfactory and easy procedure for a long colonic duplication in an infant. It provides access for monitoring the epithelium as the patient grows.

\section{References}

1. Ravitch MM. Hind gut duplication - doubling of colon and genital and urinary tracts. Annals of Surgery 1953; 137: 588-601.

2. Holcomb GW, Gheissari A, O'Neill JA, Shorter NA, Bishop HC. Surgical management of alimentary tract duplications. Annals of Surgery 1989; 209: 167-74.

3. Brown RI, Azizkhan RG. Gastrointestinal bleeding in infants and children: Meckel's diverticulum and intestinal duplication. Seminars in Pediatric Surgery 1999; 8: 202-9.

4. Bower RJ, Sieber WK, Kieswetter WB. Alimentary tract duplications in children. Annals of Surgery 1978; 188: 699-704.

5. Hickey WF. Corson JM. Squamous cell carcinoma arising in a duplication of the colon: case report and literature review of malignancy complicating colonic duplication. Cancer 1981; 47: $602-9$.

6. Adair HM, Trowell JE. Squamous cell carcinoma arising in a duplication of the small bowel. Journal of Pathology 1981; 133: 25-31.

7. Stotland BR. Stein RB. Lichtenstein GR. Advances in inflammatory bowel disease. Medical Clinics of Norh America 2000; 84: 1107-24.

\section{Creams and condoms}

One of the cornerstones of safer sex programs is correct condom use to prevent both unplanned pregnancies and the spread of sexually transmissible infections, including HIV/AIDS. However, many people are unaware that commonly used products may have an adverse effect on the protective properties of barrier contraceptives made of latex.

The products most likely to come into contact with condoms or diaphragms are personal lubricants, spermicidal preparations, personal hygiene products, hormonal preparations, acidifying agents, and preparations used for the treatment of the common vaginal infections, especially Candida. It is possible of course, that any dermatological product used on the genital area in both sexes, may have an effect on latex rubber.

Sparrow MJ. The effect of antifungal creams and pessaries on latex. Australian Prescriber 2000; 23 : 129. 